\title{
Atividade física e estresse psicológico em adolescentes: revisão sistemática
}

Physical activity and psychological stress in adolescents: systematic review

\section{Márcio Botelho Peixoto}

Programa de Pós Graduação em Epidemiologia

- Universidade Federal de Pelotas, Pelotas, Brasil.

E-mail:marcio_b_peixoto@hotmail.com

\section{Inácio Crochemore Mohnsam da Silva}

Programa de Pós Graduação em Epidemiologia

- Universidade Federal de Pelotas, Pelotas, Brasil.

E-mail:inacio_cms@yahoo.com.br

\section{Virgílio Viana Ramires}

Instituto Federal Sul-Rio-Grandense, Camaquã, Brasil.

E-mail:virgilioramires@hotmail.com

\section{Helen Denise Gonçalves}

Programa de Pós Graduação em Epidemiologia

- Universidade Federal de Pelotas, Pelotas, Brasil.

E-mail: hdgs.epi@gmail.com

\section{Resumo}

O objetivo do estudo foi revisar sistematicamente a literatura sobre a associação entre a prática de atividade física e estresse em adolescentes. Foram analisadas as bases de dados da PubMed/MEDLINE, Web of Science, SPORTDiscus, LILACS, Psyclnfo, e ADOLEC. Foi realizada a avaliação da qualidade metodológica dos artigos e todo o processo de revisão foi realizado por pares. Foram identificados 19.460 títulos, onde 176 resumos foram analisados e 104 artigos apreciados na íntegra. Ao final do processo, 11 artigos foram incluídos, cuja avaliação da qualidade metodológica apresentaram em média 9,0 (DP 1,5) pontos de 14 itens avaliados. Independente da direcionalidade, os estudos indicaram uma associação inexistente ou de pequena magnitude entre a prática de atividade física e o estresse (ou estresse psicológico) entre adolescentes investigados com delineamentos observacionais distintos. Estudos experimentais parecem demonstrar um efeito positivo da atividade física sobre o 
estresse. Maiores evidências sobre o tema ainda são necessárias, considerando diferentes aspectos do contexto familiar e do ambiente, temporalidade das associações e maior diversidade de práticas de atividade física nos estudos experimentais.

Palavras-chave: Atividade motora; Estresse psicológico; Adolescente.

\section{Abstract}

The objective of this study was to systematically review the literature on the association between the practice of physical activity and stress in adolescents. The databases were analyzed of PubMed/MEDLINE, Web of Science, SPORTDiscus, LILACS, PsycInfo, and ADOLEC. The assessment of the methodological quality of the articles and the entire process of review was performed by peers.

\section{Introdução}

A adolescência é um período da vida, marcada por um complexo processo de crescimento e desenvolvimento biopsicossocial, com experimentações e adoção de comportamentos que poderão permanecer até a idade adulta. ${ }^{1}$ Neste período da vida ocorrem mudanças psicológicas, fisiológicas e morfológicas, ${ }^{2}$ necessitando fazer escolhas e estabelecer prioridades, que, de algum modo, pode gerar
19,460 titles were identified, where 176 abstracts were analyzed and 104 articles assessed in full. At the end of the process, 11 articles were included, whose assessment of the methodological quality presented on average 9.0 (DP 1.5) 14 items points. Regardless of directionality, the studies indicated a nonexistent or small magnitude association between the practice of physical activity and stress (or psychological stress) among adolescents investigated with observational distinct designs. Experimental studies seem to demonstrate a positive effect of physical activity on stress. Major evidence on the subject are still required, considering different aspects of family and environmental context, timeliness of associations and greater diversity of practice of physical activity in experimental studies.

Keywords: Motor activity; Psychological stress; Adolescents.

estresse em distintos níveis. O estresse (também chamado de estresse psicológico) é uma resposta do organismo às demandas com reações físicas, psicológicas, mentais e hormonais frente a um evento interpretado como problemático ou desafiante. ${ }^{3,4}$

A prática de atividade física tem sido indicada como possível modo de minimizar o estresse e 
suas reações negativas ao organismo. ${ }^{5}$ No entanto, pesquisadores ${ }^{6-8}$ avaliam que o estresse psicológico como um dos fatores determinantes do comportamento fisicamente ativo, cuja determinação é bastante complexa ${ }^{9}$. Entre os adolescentes, as percepções e reações frente a eventos importantes podem interferir na adesão a comportamentos saudáveis. ${ }^{9}$ Evidências sobre a associação negativa entre o estresse psicológico e a prática de atividade física entre adultos foram demonstradas por muitos estudos. ${ }^{10}$ Pesquisas anteriores já identificaram que o estresse e a atividade física como um importante fator que pode influenciar o desenvolvimento dos adolescentes. ${ }^{11}$ Porém, os resultados de investigações com adolescentes não são consistentes ${ }^{10}$ e as consequências, a duração do estresse e seus efeitos são ainda pouco investigadas, especialmente, a longo prazo.

Este artigo objetiva revisar sistematicamente a literatura sobre a associação entre o estresse/estresse psicológico e a prática de atividade física entre adolescentes.

\section{Métodos}

Este artigo foi balizado em seu processo de revisão sistemática e de escrita de acordo com as diretrizes do PRISMA. ${ }^{12}$ A busca dos manuscritos foi realizada nas bases de dados
PubMed/ MEDLINE, LILACS, ADOLEC, Psyclnfo, SPORTDiscus e Web of Science. A definição dos descritores adotados neste processo foi baseada no sistema Medical Subject Headings (MeSH). Além deles, para que houvesse uma maior captação de artigos, foram empregados outros descritores não indexados e presentes em títulos e palavras-chave de artigos. Desse modo, foram utilizados os seguintes termos referentes à prática de atividade física, estresse e a faixa etária em estudo: "exercise", "physical effort", physical fitness", "sports", "physical activity", "motor activity", "psychological stress", "stress", "adolescent", "adolescence", "young", "youth", "teenager", "teenage", "children" e "childhood". Além disso, fez-se uso de operadores lógicos $A N D / O R$ e filtros adicionais não foram empregados. Não houve limitação de data de publicação durante a busca dos artigos.

Os critérios de inclusão dos artigos para a revisão foram a apresentação da associação entre o estresse psicológico e a atividade física - ou seja, atividade física, exercício físico e esportes - contemplando a faixa etária entre 10 e 19 anos, $^{13}$ independente do domínio avaliado para ambos. Cabe ressaltar que estudos que incluíram jovens adultos, mas cuja média de idade era menor de 19 anos, foram adicionados à revisão. A direção da associação, 
se exposição ou desfecho, não interferiu na inclusão dos manuscritos, bem como o tipo de instrumento usado.

Foram excluídos os estudos com amostras de: (a) portadores de alguma doença crônica (hipertensão, diabetes, câncer etc.); (b) portadores de necessidades especiais; (c) participantes de competições profissionais/alto rendimento. Também foram excluídos os trabalhos publicados na forma de resumo, livro, capítulo de livro e revisões sistemáticas.

O processo de busca dos artigos foi encerrado ao final de abril de 2017. Após a seleção nas bases de dados, houve a exclusão dos trabalhos duplamente captados. Todos os artigos foram inseridos em uma biblioteca no software específico para armazenar as referências bibliográficas (EndNote ${ }^{\circledR}$ ). Para a seleção final dos artigos, seguiram-se mais três etapas. No primeiro momento foi realizada a leitura dos títulos dos artigos e exclusão dos que não obedeciam aos critérios estabelecidos. No segundo, foram lidos os resumos dos artigos e selecionados os que atenderam aos critérios de inclusão e cujos resumos não foram suficientes para a tomada de decisão sobre inclusão ou exclusão. Por fim, no terceiro momento, os artigos selecionados foram analisados na íntegra, permanecendo apenas os que atenderam aos critérios de inclusão. Todas as etapas foram conduzidas de modo independente por dois revisores (MP e VR), com intuito de evitar qualquer tipo de viés de seleção dos manuscritos. Em cada etapa os revisores confrontaram os artigos e diante de uma divergência efetuaram nova leitura para decisão final. Permanecendo uma divergência, um terceiro avaliador (IS) foi consultado. Para identificar publicações não localizadas nas buscas iniciais, os artigos incluídos tiveram suas listas de referências verificadas.

Durante a análise dos artigos foram extraídos os dados referentes ao: ano de publicação e nome do periódico, delineamento do estudo, amostra, tamanho da amostra, instrumento de medida de atividade física e de estresse psicológico, além dos principais resultados.

Por fim, uma versão adaptada do checklist de Downs \& Black ${ }^{14}$ foi utilizado para avaliação da qualidade metodológica dos artigos selecionados. Foram empregados quatorze itens da escala original (Itens $01 ; 02 ; 03 ; 04 ; 06$; 07; 09; 10; $11 ; 18 ; 19 ; 20 ; 25 ; 27$; ver Tabela 1 ). Por ser um estudo de revisão, o mesmo não foi submetido ao comitê de ética.

\section{Resultados}

Na busca inicial foram encontrados 19.460 títulos em todas as bases de dados: 9.435 no PubMed/MEDLINE, 6.228 na Web of Science, 
2.474 na SPORTDisCus, 487 na LILACS, 479 na Psyclnfo e 357 na ADOLEC. Após esta seleção, 2.530 trabalhos duplicados foram excluídos através da ferramenta específica do software EndNote ${ }^{\circledR}$, permanecendo 16.930 títulos para as etapas posteriores da revisão (Figura 1).

Após a leitura dos títulos, 176 resumos foram selecionados. Destes, 104 artigos foram elegíveis para a leitura na íntegra. Entre estes, 11 trabalhos atenderam a todos os critérios de inclusão. Os principais motivos para exclusão foram: não haver testes de associação entre o estresse psicológico e atividade física $(n=37$; 41\%) e/ou não contemplar a faixa etária de interesse ( $n=42 ; 47 \%)$. Após a verificação das listas de referências dos 9 artigos, dois novos artigos foram acrescentados, somando 11 artigos inclusos nesta revisão.

$\mathrm{Na}$ avaliação dos 14 itens sobre a qualidade metodológica $^{14}$ os artigos apresentaram em média nove pontos (DP 1,5) e foram considerados, em geral, adequados para permanecerem nesta revisão. Sumariamente, em todos os estudos o objetivo estava claramente descrito. Quanto às medidas de exposição e desfecho, em nove estudos (81,8\%) elas foram consideradas acuradas (bons indicadores de validade e confiabilidade). Apenas três estudos (27,3\%) foram realizados com amostras representativas da população de interesse. Embora todos os estudos tenham realizado testes estatísticos adequados, apenas seis realizaram análises que ajustavam à associação de interesse para, ao menos, sexo e nível econômico. O resumo desta avaliação está apresentado na Tabela 1.

Entre os 11 artigos, nove foram transversais, um longitudinal e dois estudos de intervenção. 0 primeiro estudo realizado com adolescentes sobre o tema foi conduzido em 1990, com delineamento longitudinal, ${ }^{15}$ quando foram analisados os dados de 743 estudantes pertencentes a uma coorte. Os países com maior número de estudos publicados foram os Estados Unidos ${ }^{15,18,19}$ e o Brasil. ${ }^{16,20}$ No continente europeu foram realizados três estudos, ${ }^{17,}$ 21-23 enquanto que na Oceania ${ }^{24}$ e na Ásia ${ }^{25}$ um estudo em cada.

As faixas etárias estudadas divergiram entre os estudos. Yin e colaboradores (2005), ${ }^{19}$ por exemplo, consideraram adolescentes e jovens adultos (13-24 anos; média 16 anos, DP 3,3 anos) em sua amostra; Reynolds $(1990)^{15}$ entrevistou somente adolescentes, entre 14 e 16 anos; Reiner et al. $(2015)^{17}$ analisaram meninos e meninas com idade média de 14 anos e Park $(2014)^{25}$ estudou uma amostra representativa dos escolares do ensino médio (12 a 18 anos) e os trabalhos de Norris, Carroll e Cochrane $(1992)^{22}$ e Pires et al. $(2004)^{16}$ 
avaliaram estudantes adolescentes com faixas etárias similares (13 a 19 anos), também do ensino médio. Além destes, um estudo avaliou somente adolescentes meninas do ensino médio de 16 a 18 anos, ${ }^{24}$ dois entrevistaram adolescentes (13-18 anos) pertencentes a seis comunidades rurais ${ }^{21}$ e outro contemplou estudantes do 30 ano do ensino médio (17-18 anos), que pretendiam fazer prova para ingresso na universidade. ${ }^{20}$

\section{Instrumentos para avaliação do estresse}

Todos os estudos selecionados nesta revisão utilizaram questionários para a investigação do estresse psicológico. O principal objetivo dos instrumentos foi avaliar a autopercepção de estresse ou estresse psicológico. Foram abordadas diferentes perspectivas e utilizados distintos períodos recordatórios. Quatro estudos aferiram como os adolescentes habitualmente se sentiam, ${ }^{11,15,18,25}$ enquanto que outros investigaram o estresse nos últimos: sete dias, ${ }^{24}$ trinta dias, ${ }^{22}$ três meses ${ }^{17}$ e 12 meses. ${ }^{19,21}$ Apenas o estudo de Sevcikova et al. $(2001)^{23}$ não informou o período recordatório utilizado. O estudo de Araújo et al. (2012), ${ }^{20}$ além do questionário, também considerou o estresse com uma medida objetiva direta cortisol salivar.

Norris, Carroll e Cochrane (1992), ${ }^{22}$ utilizou a
Perceived Scale Stress (Escala de Estresse Percebido), ${ }^{26}$ com 14 questões. Os demais estudos utilizaram diferentes questionários, ${ }^{27-28}$ que possuíam entre 15 e 58 itens/situações investigadas, como: estresse oriundo da vizinhança, da família, dos pares interação professor/adulto; vida em casa; responsabilidade de adulto; relações amorosas, frequência escolar; conflito na escola/lazer; desempenho escolar, pressão financeira, sobrecarga escolar, tensão social e falta de tempo.

Em três destes estudos, ${ }^{29-31}$ além das perguntas relacionadas à autopercepção de estresse foram realizadas algumas perguntas sobre sintomas físicos e fisiológicos do estresse como: dificuldades em relaxar, ter tensão 'nervosa', irritabilidade e agitação, dor no estômago, aumento de sudorese, taquicardia, cansaço constante, insônia, úlcera, apatia, entre outros.

\section{Instrumentos utilizados para avaliar atividade} física

Todos os estudos observacionais utilizaram questionários com autorrelato para mensurar a atividade física. Entretanto, diferenças importantes entre os instrumentos empregados foram evidenciadas, em especial o período recordatório, a distinção ou não da intensidade (leve, moderada e vigorosa) das 
atividades físicas realizadas e o tempo de duração destas.

O menor período recordatório avaliado foi dos últimos três dias. ${ }^{16,32}$ O período mais utilizado foi o de uma semana, variando entre uma semana habitual $^{17}$ e última semana. ${ }^{15,19,22,33}$ Apenas Moljord et al. (2011) $)^{21}$ consideram as últimas quatro semanas. Feld e Shusterman $(2015)^{18}$ não informaram o tempo de recordatório utilizado para aferir a atividade física.

Assim como os domínios e intensidades avaliados em dado período de tempo expuseram importantes diferenças entre os estudos, o número de perguntas utilizadas em cada estudo foi igualmente distinto. Quatro estudos $^{17,22,24,25}$ utilizaram-se de duas a oito perguntas para mensurar o tempo de prática semanal de atividade física moderada e vigorosa. Moljord et al. (2011) utilizaram uma pergunta para avaliar exclusivamente as atividades físicas vigorosas realizadas, por pelo menos 20 minutos/dia, nas últimas quatro semanas. Reynolds et al. (1990) ${ }^{15}$ avaliaram, com uma escala Likert, o autorrelato da frequência das atividades físicas com duração de pelo menos 20 minutos consecutivos em uma semana. Em outros dois estudos ${ }^{18,19}$ a atividade física foi medida com uma questão sobre a frequência de dias/semana em que foram praticadas, sem considerarem a intensidade e o tempo gasto em cada uma delas. Sevcikova et al. (2001) ${ }^{23}$ compilaram as atividades esportivas entre escolares através do resultado da frequência, da intensidade e do tempo de atividades, mas não mencionaram as perguntas usadas.

\section{Intervenções}

Os dois estudos que realizaram intervenções com atividade física para avaliar o efeito o estresse psicológico, utilizaram sessões de atividades físicas moderadas e vigorosas. A intervenção realizada por Araújo et al. (2012) avaliou sessões de exercícios físicos aeróbios de intensidade moderada e exercícios resistidos, realizados em circuitos com duração aproximada de 60 minutos, duas vezes por semana durante dez semanas. Norris, Carroll e Cochrane $(1992)^{22}$ realizaram uma intervenção com atividade física aeróbia com duas intensidades (alta: 70 - 75\% da frequência cardíaca máxima; moderada: $60-65 \%$ da frequência cardíaca máxima), ${ }^{34}$ com duas sessões por semana de 25 a 30 minutos durante 10 semanas.

\section{Resultados dos estudos observacionais}

O Quadro 1 resume todos os sete estudos transversais, cujo o desfecho foi o estresse psicológico e a exposição a atividade física. 
Nestes, os participantes eram adolescentes escolares (10 até 18 anos), com amostras variando de 147 até mais de 73 mil estudantes. Uma grande amplitude de categorização de faixa etária foi observada.

Os principais resultados ressaltaram a ausência ou a pequena magnitude da associação entre a prática de atividade física e o estresse psicológico. Dois estudos ${ }^{24,25}$ não encontraram associação, sendo que um deles mostrou que os adolescentes fisicamente ativos tinham menor probabilidade de serem classificados com estresse psicológico acima da média. Entretanto, a medida de efeito encontrada foi pequena, com uma razão de odds de $0,89-I C 95 \%=0,86-0,93$. $^{25}$ Outros quatro estudos, ${ }^{18,19,21,22,25}$ destacaram coeficiente de correlação muito fraco ou fraco, variando entre $-0,08 a-0,23$.

Já o estudo realizado com estudantes, residentes na Eslováquia, ${ }^{23}$ demonstrou que meninos que atingiram a recomendação de 300 minutos de atividade física semanais relataram menor carga de estresse quando comparados aos meninos que não atingiram a recomendação. Entre as meninas, esta diferença não foi observada. Importante destacar que, neste estudo, nenhuma medida de efeito foi apresentada, apenas diferenças entre os grupos.
Entre os três estudos observacionais, ${ }^{15-17}$ que utilizaram a atividade física como desfecho (Quadro 2), nenhum estudo foi realizado com amostra representativa do público alvo, mas todos foram desenvolvidos com escolares, a exemplo dos apresentados do Quadro 1.

Nos estudos transversais realizados por Pires et al. ${ }^{16}$ e Reiner et al. ${ }^{17}$ não foram apresentadas nas análises as medidas de efeito. Contudo, no estudo de Pires et al. ${ }^{16}$ evidenciou maior número de horas em atividades físicas entre adolescentes que não relataram sintomas de estresse. Além disso, o grupo classificado pelo autor como com estresse adequado à saúde teve maior participação em atividades de esforço muito intenso quando comparado ao grupo com estresse prejudicial $(p<0,05)$. 0 estudo de Reiner et al. ${ }^{17}$ apontou uma diminuição no tempo gasto com atividades físicas moderadas e vigorosas quando o estresse era oriundo da sobrecarga escolar. No único estudo longitudinal desta revisão, ${ }^{15}$ os autores não encontraram correlação entre a prática da atividade física e o estresse em nenhum momento investigado.

\section{Resultados dos estudos experimentais}

Dois estudos experimentais ${ }^{20,22}$ examinaram o efeito da atividade física sobre o estresse (Quadro 3). Ao avaliarem o efeito da atividade 
física moderada (aeróbia e exercícios de fortalecimento muscular $)^{22}$ e atividades aeróbias de alta intensidade ${ }^{20}$ sobre o estresse, encontraram reduções no estresse percebido entre os participantes do grupo intervenção, quando comparados os períodos pré e pósintervenção. No estudo de Araújo et al. (2012) encontrou que os adolescentes que não praticaram atividade física durante o período estudado tiveram um aumento da sua percepção de estresse.

\section{Discussão}

A presente revisão sistemática da literatura evidenciou que a associação entre estresse (ou estresse psicológico) e atividade física são ainda inconsistentes. Independente da direcionalidade e dos domínios de atividade física e estresse avaliados, os estudos indicaram uma associação inexistente ou de pequena magnitude entre a prática de atividade física e o estresse entre adolescentes investigados com delineamentos observacionais distintos. Os estudos transversais, intrinsecamente limitados para estabelecer uma relação causal, não foram suficientes para evidenciar a relação entre o estresse e a atividade física. Apenas três deles confirmaram a associação de interesse. Os dois estudos experimentais analisados indicaram um efeito positivo da prática sobre o estresse percebido. ${ }^{20,22}$
Alguns aspectos metodológicos e analíticos podem justificar a falta de evidências. Verificouse uma grande variabilidade de instrumentos empregados para avaliar tanto a atividade física quanto o estresse. Diferentes domínios de avaliação da atividade física e do estresse foram encontrados nos estudos. Apesar do tipo de estresse avaliado ser o mesmo (estresse psicológico percebido ou estresse), as categorias analisadas e as distintas pontuações dificultaram uma comparação mais precisa entre alguns estudos. Desta forma, nossas análises foram norteadas para averiguar se houve associação ou não entre esses fatores.

Poucos estudos ${ }^{17,21,25}$ desta revisão foram realizados com amostras representativas da população alvo, igualmente para faixa etária de interesse, onde existe a necessidade de estudos com faixas etárias homogêneas. Este aspecto pode fazer com que os adolescentes investigados tenham características diferentes daqueles que não foram comtemplados, como por exemplo, menor exposição ao estresse. Além disso, pequeno tamanho das amostras pode ter limitado o poder das análises e impedido estratificações necessárias.

Entre os 11 incluídos, seis realizaram análises ajustadas e/ou estratificadas para sexo e nível socioeconômico. Sabe-se que, em geral, as meninas relatam maiores níveis de estresse e 
que a prática de atividade física é maior entre os meninos, quando ambos são comparados ao sexo contrário. ${ }^{10,35}$ Todavia, assim como o sexo, fatores socioeconômicos também podem interagir na associação entre atividade física e estresse, potencializando ou inibindo essa associação entre adolescentes de diferentes classes socioeconômicas.

Acredita-se ainda que não apenas as características individuais possam promover ou inibir a prática de atividade física e a percepção do estresse, mas que os aspectos do ambiente físico, econômico e social têm um peso a ser considerado na avaliação das mudanças de trajetória ou padrão da atividade física na população adolescente. ${ }^{36}$

Como a plausibilidade existente na associação entre atividade física e estresse é comprovada fisiologicamente - o exercício aeróbio estimula a liberação de endorfinas ${ }^{37}$ e proporciona uma sensação de bem-estar físico e mental ${ }^{38}$ - era esperado encontrar resultados semelhantes e positivos para a associação entre atividade física e estresse também entre adolescentes. Neste sentido, algumas características intrínsecas ao tema em pesquisa e aos estudos desta revisão também podem ter se refletido nos achados. Por exemplo, atividades físicas realizadas no deslocamento ou no tempo livre estão expostas à insegurança na cidade, ao clima desfavorável, a estrutura ofertada, entre outros fatores contextuais, afetando a percepção de estresse e a continuidade ou intensidade desta prática. Como os estudos não contemplaram outras especificidades importantes da atividade física e possíveis fontes do estresse, ${ }^{10}$ também limitaram suas análises ao não explorar os fatores sinergistas da atividade física (clima/estação do ano do período das avaliações ou de provas/exames).

Entende-se que para a avaliação da atividade física, os usos de questionários padronizados que meçam diferentes intensidades das atividades assim como suas durações auxiliariam na compreensão da relação da atividade física com o estresse. Instrumentos empregados para avaliar o estresse podem analisar diferentes fontes dele a fim de identificar e esclarecer suas relações específicas com um comportamento ativo. Tanto na mensuração do estresse quanto da prática de atividade física, o uso de medidas objetivas (como cortisol salivar e acelerometria) acrescentaria qualidade na avaliação do componente biológico destes comportamentos, evitando os reconhecidos vieses de informação. Todavia, utilizar somente medidas objetivas não fornecerá a distinção das fontes de estresse e tampouco sobre os domínios da prática de atividade física, 
aspectos importantes para avaliar as associações. Logo, a combinação de métodos objetivos e subjetivos é metodologicamente mais adequada, devendo ser incentivada e estudos futuros.

\section{Conclusão}

A partir do discutido, existe a necessidade de incluir diversas interações de múltiplos níveis, adaptações, ações concorrentes e consequências não intencionais que podem influenciar a prática de atividade física e o estresse. Maiores evidências, considerando uma uniformidade de instrumentos e avaliações, sobre o tema ainda são necessárias para a população adolescente, especialmente para contemplar diferentes aspectos do contexto familiar e no qual os jovens estão inseridos (como a escola), a temporalidade das associações e a diversidade de práticas de atividade física usando estudos experimentais.

\section{Referências}

\footnotetext{
${ }^{1}$ Azevedo M, Araújo C, Silva M, Hallal P. Tracking of physical activity from adolescence to adulthood: a population-based study. Rev saúde pública. 2007;41(1):69-75.

${ }^{2}$ Department of Health and Human Services (US). Physical activity guidelines for Americans midcourse report: strategies to increase physical activity among youth. US Department of Health and Human Services; 2012.

${ }^{3}$ Groër M, Thomas S, Schoffner D. Adolescence stress and coping: a longitudinal study. Research in Nursing and Health. 1992;15:209-217.

${ }^{4}$ Lipp M, Malagris L. O stress emocional e seu tratamento. In: Rangé, B. Psicoterapias cognitivo-comportamentais. São Paulo: Artmed; 2001.

${ }^{5}$ Penedo F, Dahn J. Exercise and well-being: a review of mental e physical health benefits associated with physical activity. Curr Opin Psychiatr. 2005;18(2):189-193.

${ }^{6}$ Johansson G, Johnson J, Hall E. Smoking and sedentary behavior as related to work organization. Soc Sci Med. 1991;32(7):837-846.

${ }^{7}$ Burdette A, Hill T. An examination of processes linking perceived neighborhood disorder and obesity. Soc Sci Med. 2008;67(1):38-46.

${ }^{8}$ Fan Y, Das K, Chen Q. Neighborhood green, social support, physical activity, and stress: assessing the cumulative impact. Health Place. 2011;17(6):1202-1211.

${ }^{9}$ Bauman A, Reis R, Sallis J, Wells J, Loos R, Martin B. Correlates of physical activity: why are some people physically active and others not? The Lancet. 2012;380:258-271.

${ }^{10}$ Stults-Kolehmainen M, Sinha R. The effects of stress on physical activity and exercise. Sports Med. 2014; 44(1):81-121.

${ }^{11}$ Haugland S, Wold B, Torsheim T. Relieving the pressure? the role of physical activity in the relat'onship between schoolrelated stress and adolescent health complaints. Research Quarterly Exercise Sport. 2003;74(2):127.

${ }^{12}$ Moher D, Liberati A, Tetzlaff J, Altman D. Preferred reporting items for systematic reviews and meta-analyses: the PRISMA statement. PLoS Med. 2009;6(7).

${ }^{13}$ World Health Organization. Young people's health - a challenge for society report of a who study group on young people and health for all. Technical Report Series 731 Geneva: WHO. 1986.
} 
${ }^{14}$ Downs S, Black N. The feasibility of creating a checklist for the assessment of the methodological quality both of randomised and non-randomised studies of health care interventions. J Epidemiol Community Health. 1998;52:377-84.

${ }^{15}$ Reynolds K, Killen J, Bryson S, Maron D, Taylor C, Maccoby N, et al. Psychosocial predictors of physical activity in adolescents. Prev Med. 1990;19(5):541-51.

${ }^{16}$ Pires E, Duarte M, Pires M, Souza G. Hábitos de atividade física e o estresse em adolescentes de Florianópolis - SC, Brasil. R bras Ci e Mov. 2004;12(1):51-6.

${ }^{17}$ Reiner M, Niermann C, Krapf F, Woll A. Stress: personal matter or family affair? intra-and inter-individual relationships between stress, physical activity, sedentary behavior, and nutrition. Int J Child Youth Family Stud. 2015;6(1):68-92.

${ }^{18}$ Feld L, Shusterman A. Into the pressure cooker: student stress in college preparatory high schools. J Adolesc. 2015; 14(41):31-42.

${ }^{19}$ Yin Z, Davis C, Moore J, Treiber F. Physical activity buffers the effects of chronic stress on adiposity in youth. Ann Behav Med. 2005;29(1):29-36.

${ }^{20}$ Araújo M, França N, Madeira F, Sousa Júnior, Silva G, Silva E, et al. Efeitos do exercício físico sobre os níveis de estresse em vestibulandos de Teresina-PI. R Bras Ci e Mov. 2012;20(3):14-26.

${ }^{21}$ Moljord I, Moksnes U, Eriksen L, Espnes G. Stress and happiness among adolescents with varying frequency of physical activity. Percept Mot Skills. 2011;Oct(113):2.

${ }^{22}$ Norris R, Carroll D, Cochrane R. The effects of physical activity and exercise training on psychological stress and well-being in an adolescent population. J Psychosom Res. 1992; 36(1):55-65.

${ }^{23}$ Sevcikova L, Stefanikova Z, Jurkovicova J, Ruzanska S, Sabolova M, Aghova L. Stress and health-related behaviour, personality characteristics and blood pressure in older school children. Bratislava Med J. 2001;102(9):420-423.

${ }^{24}$ Shepherd D, Krägeloh C, Ryan C, Schofield G. Psychological well-being, self-reported physical activity levels, and attitudes to physical activity in a sample of New Zealand adolescent females. Psychology. 2012;3(6):447-53.

${ }^{25}$ Park S. Associations of physical activity with sleep satisfaction, perceived stress, and problematic Internet use in Korean adolescents. BMC Public Health. 2014;14.

${ }^{26}$ Cohen S, Kamark T, Mermelstein R. A global measure of perceived stress. J. Health Soc. Behav. 1983;24(4):385-96.

${ }^{27}$ Ewart C. The Adolescent Resource Challenges Scale (ARCS): Reliability and validity. Unpublished manuscript Johns Hopkins University; 1993.

${ }^{28}$ Richartz A, Hoffmann K, Sallen J. Scales for chronic stress in childhood. Children in professional sports - Chronic strain and protective resources. Schorndorf: Hofmann. 2009.p.77-80; 334-338.

${ }^{29}$ Lovibond S, Lovibond P. Manual for the depression anxiety stress scales. 2nd ed. Sydney: Psychology Foundation. 1995.

${ }^{30}$ Tricoli V, Lipp M. ESA: Escala de stress para adolescentes. São Paulo: Casa do Psicólogo. 2005.

${ }^{31}$ Lipp M, Guevara A. Validação empírica do estresse. Est Psic. 1994;11(3):43-49.

32 Pires E, De Bem M, Pires M, Barros M, Duarte M, Nahas M. Reproducibility and validity of the 3 DPAR physical activity questionnaire in a sample of brazilian adolescents. Med Sci Sports Exerc. 2001;33(5):S144.

${ }^{33}$ Mackay L, Schofield G, Schluter P. Validation of self-report measures of physical activity: A case study using the New Zealand physical activity questionnaire. Res Quart Exercise Sport. 2007;78:189-96.

${ }^{34}$ Cullum R, Mowbray L. The English YMCA Guide to Exercise to Music. London: Pelham Books; 1988.

${ }^{35}$ Hallal PC, Andersen LB, Bull FC, Guthold R, Haskell W, Ekelund U. Global physical activity levels: surveillance progress, pitfalls, and prospects. The Lancet. 380(9838):247-257.

${ }^{36}$ Sallis J, Hovell M, Hofstetter C, Elder J, Hackley M, Caspersen C, et al. Distance between homes and exercise facilities related to frequency of exercise among San Diego residents. Public Health Rep. 1990; 105(2):179-185.

${ }^{37}$ Boecker H, Sprenger T, Spilker M, Henriksen G, Koppenhoeffer M, Wagner K, et al. The runners' high: opioidergic mechanisms in the human brain. Cereb Cort. 2008;18:2523-2531.

${ }^{38}$ Stefano G, Goumon Y, Casares F, Cadet P, Fricchione G, Rialas C, et al. Endogenous morphine. Trends Neurosci. 2000; 23(9):436-442. Review. PubMed PMID: 10941194.

${ }^{39}$ Moksnes U, Byrne D, Mazanov J, Espnes G. Adolescent stress: evaluation of the factor structure of the Adolescent Stress Questionnaire (ASQ-N). Scand J Psychol. 2010;51:203-209. 
${ }^{40}$ Brown J, Lawton M. Stress and well-being in adolescence: the moderating role of physical exercise. J Human Stress. 1986; 12(3):125-131.

${ }^{41}$ Anderson R. Stretching. London: Pelham Books. 1989.

\section{Anexo}

Figura 1: Fluxograma da seleção de artigos da revisão sistemática.

Figure 1: Flowchart of the selection of articles from the systematic review.

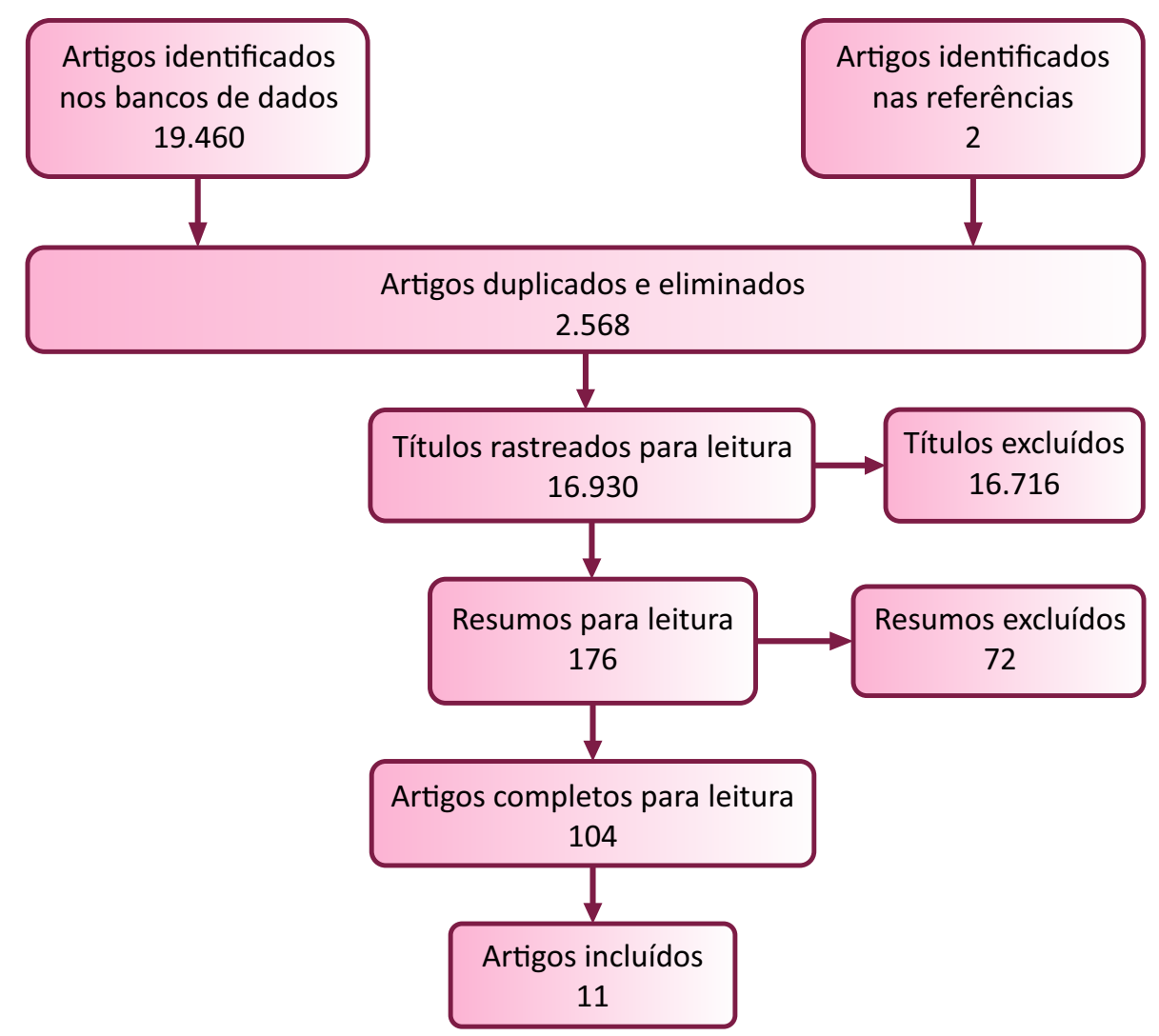


Tabela 1: Versão adaptada de Downs \& Black $(1998)^{14}$ para a avaliação da qualidade metodológica dos estudos incluídos ( $\mathrm{N}=11)$.

\begin{tabular}{|c|c|c|}
\hline Item & Critérios* & $\mathbf{N}(\%)$ \\
\hline & Descrição & \\
\hline 01 & Hipótese/objetivo do estudo estão claramente descritos? & $11(100)$ \\
\hline 02 & $\begin{array}{l}\text { Os desfechos a serem medidos estão claramente descritos na introdução ou na seção } \\
\text { de métodos? }\end{array}$ & $10(90,9)$ \\
\hline 03 & As características dos indivíduos incluídos no estudo estão claramente descritas? & $9(84,6)$ \\
\hline 04 & As exposições de interesse estão claramente descritas? & $11(100)$ \\
\hline 06 & Os principais achados do estudo são claramente descritos? & $10(90,9)$ \\
\hline 07 & $\begin{array}{l}\text { O estudo proporciona estimativas da variabilidade aleatória dos dados dos principais } \\
\text { achados? }\end{array}$ & $8(72,7)$ \\
\hline 09 & As características dos participantes perdidos foram descritas? & $3(27,3)$ \\
\hline \multirow[t]{2}{*}{10} & $\begin{array}{l}\text { Os intervalos de confiança de } 95 \% \text { e/ou valores de } p \text { foram relatados para os principais } \\
\text { desfechos, exceto quando o valor } p \text { foi menor que } 0,001 \text { ? }\end{array}$ & $7(63,6)$ \\
\hline & Validade externa & \\
\hline \multirow[t]{2}{*}{11} & $\begin{array}{l}\text { Os sujeitos chamados para participar do estudo foram representativos de toda a } \\
\text { população de onde foram recrutados? }\end{array}$ & $3(27,3)$ \\
\hline & Validade interna: viés & \\
\hline 18 & Os testes estatísticos utilizados para avaliar os principais desfechos foram apropriados? & $11(100)$ \\
\hline 19 & As exposições foram confiáveis, ou seja, sem erro de classificação? & $9(81,8)$ \\
\hline 20 & As medidas dos principais desfechos foram acuradas (válidas e confiáveis)? & $9(81,8)$ \\
\hline 25 & $\begin{array}{l}\text { Houve um ajuste adequado dos fatores de confusão nas análises a partir das quais os } \\
\text { principais achados foram tirados? }\end{array}$ & $6(54,5)$ \\
\hline 27 & $\begin{array}{l}\text { O estudo tem poder suficiente para detectar um efeito clinicamente importante } \\
\text { quando o valor de p ("probability value") para uma diferença que é devida ao acaso é } \\
\text { inferior a } 5 \% \text { ? }\end{array}$ & $6(54,5)$ \\
\hline
\end{tabular}


Quadro 1: Sumário dos estudos observacionais (transversais) da relação entre estresse psicológico e atividade física.

\begin{tabular}{|c|c|c|c|c|}
\hline $\begin{array}{l}\text { Autor } \\
\text { Ano }\end{array}$ & $\begin{array}{l}\text { N; Amostra; } \\
\text { Idade }\end{array}$ & $\begin{array}{l}\text { Medida de Estresse } \\
\text { (definição operacional) }\end{array}$ & $\begin{array}{c}\text { Medida de AF } \\
\text { (definição operacional) }\end{array}$ & Principais resultados \\
\hline $\begin{array}{c}\text { Feld e } \\
\text { Shusterman, } \\
2015^{18}\end{array}$ & $\begin{array}{l}\text { 333; Escola } \\
\text { pública e } \\
\text { privada do } 9 \text { o } \\
\text { ao } 12 \text { ano }\end{array}$ & $\begin{array}{l}\text { Autoperceção de estresse } \\
\text { através de uma pergunta: } \\
\text { (média de pontos) }\end{array}$ & $\begin{array}{l}\text { Dias por semana em que } \\
\text { pratica atividades físicas } \\
\text { (média e DP) }\end{array}$ & $\begin{array}{c}\text { Fraca correlação inversa } \\
\text { entre o nível de estresse e } \\
\text { frequência das atividades } \\
\text { físicas } r=-0,13\end{array}$ \\
\hline $\begin{array}{l}\text { Moljord et } \\
\text { al., } 2011^{21}\end{array}$ & $\begin{array}{l}1.508 ; \\
\text { Estudantes de } \\
\text { ensino médio; } \\
\text { 13-18 anos }\end{array}$ & $\begin{array}{l}\text { Versão norueguesa do } \\
\text { Questionário de Estresse } \\
\text { para Adolescentes } \\
\text { (baixo, moderado e alto } \\
\text { estresse) }\end{array}$ & Prática de atividade física & $\begin{array}{l}\text { Fraca correlação inversa } \\
\text { entre atividade física } \\
\text { vigorosa e estresse } \\
\text { (Meninas: } r=-0,13 ; \\
\text { Meninos: } r=-0,08 \text { ) }\end{array}$ \\
\hline $\begin{array}{l}\text { Norris, } \\
\text { Carroll, e } \\
\text { Cochrane, } \\
1992^{22}\end{array}$ & $\begin{array}{l}147 \\
\text { Estudantes do } \\
\text { ensino médio; } \\
\text { 13-17 anos; }\end{array}$ & $\begin{array}{l}\text { Escala de estresse } \\
\text { percebido }{ }^{26} \text { com } 14 \text { itens } \\
\text { (média de pontos e DP) }\end{array}$ & $\begin{array}{l}\text { Tempo habitual gasto } \\
\text { por semana em } \\
\text { atividades físicas e a } \\
\text { intensidade }^{40}\end{array}$ & $\begin{array}{l}\text { Fraca correlação inversa } \\
(r=-0,23 ; p<0,01) \text { entre } \\
\text { atividade física e estresse }\end{array}$ \\
\hline Park, $2014^{25}$ & $\begin{array}{c}73.238 ; \\
\text { Amostra } \\
\text { representativ } \\
\text { a ;12-18 anos }\end{array}$ & $\begin{array}{l}\text { Estresse habitual, } \\
\text { avaliado com uma } \\
\text { pergunta e resposta } \\
\text { (estresse percebido } \\
\text { abaixo e acima da média) }\end{array}$ & $\begin{array}{l}\text { Atividade física } \\
\text { moderada e vigorosa, } \\
\text { avaliado por meio de } \\
\text { duas perguntas } \\
\text { elaboradas pelo autor }\end{array}$ & $\begin{array}{l}\text { Adolescentes } \\
\text { considerados fisicamente } \\
\text { ativos apresentaram uma } \\
\text { proteção de } 11 \% \text { (RO= } \\
0,89 \text { IC } 95 \%=0,86-0,93 ; \\
p=<0.001)\end{array}$ \\
\hline $\begin{array}{l}\text { Sevcikova et } \\
\text { al., } 2001^{23}\end{array}$ & $\begin{array}{l}\text { 213; } \\
\text { Estudantes; } \\
\text { 10-14 anos }\end{array}$ & $\begin{array}{l}\text { Estresse na escola e em } \\
\text { casa (Frequentemente ou } \\
\text { raramente estressado) }\end{array}$ & $\begin{array}{c}\text { Frequência, intensidade } \\
\text { e tempo de exercícios } \\
\text { das crianças na escola } \\
\text { durante a semana }\end{array}$ & $\begin{array}{l}\text { Os meninos ativos } \\
\text { relataram uma menor } \\
\text { carga de estresse }(p=0,02)\end{array}$ \\
\hline $\begin{array}{l}\text { Shepherd et } \\
\text { al., } 2012^{24}\end{array}$ & $\begin{array}{l}148 ; \\
\text { Estudantes } \\
\text { meninas (16- } \\
18 \text { anos) }\end{array}$ & $\begin{array}{l}\text { Escala de Depressão, } \\
\text { Ansiedade e Estresse } \\
\text { (média e DP) }\end{array}$ & $\begin{array}{l}\text { Questionário de } \\
\text { Atividade Física da Nova } \\
\text { Zelândia }{ }^{33} \text { (relativamente } \\
\text { inativos; ativos e muito } \\
\text { ativos }\end{array}$ & $\begin{array}{l}\text { Não foi encontrada } \\
\text { diferenças } \\
\text { estatisticamente } \\
\text { significativas }\end{array}$ \\
\hline $\begin{array}{l}\text { Yin et al., } \\
2005^{19}\end{array}$ & $\begin{array}{c}\text { 303; } \\
\text { Adolescentes; } \\
\text { 13-24 anos }\end{array}$ & $\begin{array}{l}\text { Estresse pessoal nos } \\
\text { últimos } 12 \text { meses } \\
\text { (média e desvio padrão } \\
\text { da escala likert) }\end{array}$ & $\begin{array}{l}\text { Número de dias de } \\
\text { atividade físicas na } \\
\text { semana (média e DP) }\end{array}$ & $\begin{array}{l}\text { Correlação muito fraca } \\
\text { entre a atividade física e o } \\
\text { estresse pessoal }(r=0,08)\end{array}$ \\
\hline
\end{tabular}


Quadro 2: Sumário dos estudos observacionais da relação entre atividade física e estresse psicológico.

\begin{tabular}{|c|c|c|c|c|}
\hline $\begin{array}{l}\text { Autor } \\
\text { Ano }\end{array}$ & $\begin{array}{l}\text { Delineamento } \\
\text { N; Amostra; Idade }\end{array}$ & $\begin{array}{c}\text { Medida de Estresse } \\
\text { (definição operacional) }\end{array}$ & $\begin{array}{c}\text { Medida de AF } \\
\text { (definição operacional) }\end{array}$ & Principais resultados \\
\hline $\begin{array}{l}\text { Pires et al., } \\
2004^{16}\end{array}$ & $\begin{array}{l}\text { Transversal } \\
\text { 754; Estudantes } \\
\text { do de escolas } \\
\text { estaduais, } \\
\text { particulares e } \\
\text { federais; } 15 \text { a } 19 \\
\text { anos }\end{array}$ & $\begin{array}{l}\text { Inventário de sintomas } \\
\text { de estresse }{ }^{31} \text { nas } \\
\text { últimas } 24 \text { horas, } \\
\text { última semana e no } \\
\text { último mês. (estresse } \\
\text { adequado ou } \\
\text { prejudicial à saúde) }\end{array}$ & $\begin{array}{l}\text { Recordatório de três dias } \\
\text { de atividade física } \\
\text { (tempo e níveis de }^{32} \\
\text { esforço em atividades } \\
\text { físicas em quatro } \\
\text { categorias: Leve, } \\
\text { moderado; intenso e } \\
\text { muito intenso) }\end{array}$ & $\begin{array}{l}\text { O grupo estresse adequado a } \\
\text { saúde, apresentou maior } \\
\text { número de horas em atividades } \\
\text { físicas e maior participação em } \\
\text { atividades de esforço muito } \\
\text { intenso que o grupo com } \\
\text { estresse prejudicial ( } p<0,05 \text { ) } \\
\text { No grupo na fase de alerta, } \\
\text { apresentou um maior percentual } \\
\text { de tempo despendido em } \\
\text { atividades físicas (níveis } \\
\text { moderado, intenso e muito } \\
\text { intenso), comparado com a fase } \\
\text { de exaustão }\end{array}$ \\
\hline $\begin{array}{l}\text { Reynolds } \\
\text { et al., } \\
1990^{15} \\
\text { Longitudin } \\
\quad \text { al }\end{array}$ & $\begin{array}{l}\text { Longitudinal } \\
\text { 374; Escolares } \\
\text { do } 10 \text { o ano; } 14 \text { - } \\
16 \text { anos }\end{array}$ & $\begin{array}{l}\text { Foi avaliado no } \\
\text { Baseline e } 16 \text { meses } \\
\text { após a quantidade das } \\
\text { tensões geradas por } \\
\text { uma série de } 15 \text { ( } \\
\text { escala likert - média e } \\
\text { DP) }\end{array}$ & $\begin{array}{c}\text { Autorrelato da } \\
\text { frequência a partir de } \\
\text { uma lista de } 19 \text { itens } \\
\text { com atividades físicas }{ }^{15} \\
\text { (1-3 vezes/ mês; } 1-2 \\
\text { vezes/sem; } 3-5 \\
\text { vezes/sem e todos os } \\
\text { dias) }\end{array}$ & $\begin{array}{l}\text { Não houve correlação entre } \\
\text { frequência das atividades físicas } \\
\text { e estresse percebido em } \\
\text { meninos (baseline } r=-0,02 \text {; e } \\
\text { aos } 16 \text { meses } r=0,03 ; \text { ) e } \\
\text { meninas (baseline } r=-0,09 \text {; e } \\
\text { aos } 16 \text { meses } r=-0,03 \text { ) }\end{array}$ \\
\hline $\begin{array}{l}\text { Reiner et } \\
\text { al., } 2015^{17} \\
\text { Transversal }\end{array}$ & $\begin{array}{l}\text { Transversal } \\
\text { 211; Alunos de } \\
11 \text { escolas } \\
\text { secundárias; } \\
\text { Idade média de } \\
\text { 14,1 (DP } 1,4 \text { ) }\end{array}$ & $\begin{array}{l}\text { Escala de Estresse } \\
\text { Crônico durante a } \\
\text { Infância }{ }^{28} \text { (escala Likert } \\
\text { de cinco pontos) }\end{array}$ & $\begin{array}{c}\text { Atividade física } \\
\text { moderada e vigorosa } \\
\text { durante uma semana } \\
\text { normal e durante a } \\
\text { última semana } \\
\text { (intensidades das } \\
\text { atividades foi calculado o } \\
\text { gasto energético (MET)) }\end{array}$ & $\begin{array}{l}\text { Não foi relacionado ao estresse } \\
\text { global percebido. No entanto, } \\
\text { adolescentes gastaram menos } \\
\text { tempo em atividades de } \\
\text { moderada a vigorosa se eles } \\
\text { estavam estressados devido à } \\
\text { sobrecarga de escola }(-0,102 \\
\text { p }<0,05)\end{array}$ \\
\hline
\end{tabular}


Quadro 3: Sumário dos estudos experimentais sobre atividade física e o estresse psicológico.

\begin{tabular}{|c|c|c|c|c|}
\hline $\begin{array}{l}\text { Autor } \\
\text { Ano }\end{array}$ & $\begin{array}{l}\text { N; Amostra; } \\
\text { Idade }\end{array}$ & $\begin{array}{c}\text { Medida de Estresse } \\
\text { (definição operacional) }\end{array}$ & $\begin{array}{c}\text { Medida de AF } \\
\text { (definição operacional) }\end{array}$ & Principais resultados \\
\hline $\begin{array}{l}\text { Araújo et } \\
\text { al., } 2012^{20}\end{array}$ & $\begin{array}{l}\text { 71; (39 Grupo } \\
\text { Experimental; } \\
32 \text { Grupo } \\
\text { Controle); } \\
\text { Estudantes } \\
\text { avaliados } \\
\text { como } \\
\text { estressados e } \\
\text { inativos do } 30 \\
\text { ano do ensino } \\
\text { médio; } 17-18 \\
\text { anos }\end{array}$ & $\begin{array}{l}\text { Escala de Estresse para } \\
\text { Adolescentes }{ }^{30} \text { com } 44 \\
\text { itens (alerta, } \\
\text { resistência, quase } \\
\text { exaustão e exaustão) e } \\
\text { sintomas do estresse } \\
\text { (sintomas psicológicos, } \\
\text { cognitivos, fisiológicos e } \\
\text { interpessoais). Análise } \\
\text { do Cortisol salivar } \\
\text { (média e DP) }\end{array}$ & $\begin{array}{l}\text { Programa de exercício físico } \\
\text { moderado } \\
\text { Intervenção: Sessões de } 60 \\
\text { minutos, duas vezes por } \\
\text { semana, durante } 10 \text { semanas } \\
\text { Controle: não participaram } \\
\text { do fator experimental }\end{array}$ & $\begin{array}{l}\text { Redução dos escores médios } \\
\text { das fases e sintomas do estresse } \\
\text { entre os adolescentes do grupo } \\
\text { experimental, tanto em } \\
\text { meninas, quanto meninos (Fase } \\
\text { - } p=0,001 \text {; Sintoma - } p=0,001 \text { ) } \\
\text { Redução do Cortisol no grupo } \\
\text { experimental (Pré-intervenção } \\
0,34 \pm 0,15 \text { e no Pós- } \\
\text { intervenção } 0,16 \pm 0,09-p= \\
0,001 \\
\text { No Grupo controle houve um } \\
\text { aumento dos escores médios } \\
\text { das fases e sintomas do estresse } \\
\text { entre meninos e meninas (Fase - } \\
p=0,024 ; \text { Sintoma - } p=0,001 \text { ). } \\
\text { Também houve aumento do } \\
\text { cortisol ( } p=0,001 \text { ) }\end{array}$ \\
\hline $\begin{array}{l}\text { Norris, } \\
\text { Carroll e } \\
\text { Cochrane, } \\
1992^{22}\end{array}$ & $\begin{array}{l}\text { 72; } \\
\text { Estudantes do } \\
\text { ensino médio; } \\
\text { 13-17 anos }\end{array}$ & $\begin{array}{c}\text { Escala de estresse } \\
\text { percebido }{ }^{26} \text { com } 14 \\
\text { itens (média de pontos } \\
\text { geral da escala e desvio } \\
\text { padrão) }\end{array}$ & $\begin{array}{l}\text { Atividade física aeróbia ( } 2 \\
\text { sessões/semana; } 30 \text { minutos, } \\
\text { durante } 10 \text { semanas) } \\
\text { Diferentes intensidades: } \\
\text { (Alta: } 70-75 \% \text { da frequência } \\
\text { cardíaca máxima; Moderada: } \\
60-65 \% \text { da frequência } \\
\text { cardíaca máxima }{ }^{34} \text { ) } \\
\text { Grupo flexibilidade }{ }^{41} \text {; Grupo } \\
\text { controle: não realizou } \\
\text { atividade }\end{array}$ & $\begin{array}{l}\text { Após a intervenção foi } \\
\text { encontrada uma redução } \\
\text { estatisticamente significativa no } \\
\text { estresse percebido no grupo } \\
\text { que realizou atividade física de } \\
\text { alta intensidade ( } p=0,01 \text { ). Não } \\
\text { foram observadas diferenças } \\
\text { estatisticamente significativas } \\
\text { nos grupos de intervenção de } \\
\text { atividade física moderada, } \\
\text { flexibilidade e no grupo controle }\end{array}$ \\
\hline
\end{tabular}

